\title{
SUR PROTAGORAS 351C4-5 ET 352B3-C2
}

Marco ZINGANO

Universidade de São Paulo

Le Protagoras est sûrement l'un des plus beaux dialogues de Platon. Il est traditionnellement placé parmi les dialogues socratiques de Platon, en compagnie, entre autres, du Lachès, de l'Euthyphron et du Charmide. Ces derniers dialogues examinent une vertu en particulier (respectivement : le courage, la piété, la tempérance), alors que, dans le Protagoras, il s'agit de comprendre non pas une certaine vertu, mais l'unité même des vertus. Par là, il se distingue déjà de ces dialogues, tout en restant dans le cadre des rencontres socratiques caractéristiques de la première période de la production intellectuelle de Platon. Mais il a aussi une ampleur littéraire qui le place dans une position privilégiée. En effet, c'est un dialogue complexe, avec un préambule sophistiqué - l'arrivée à la maison de Callias devenue célèbre avec cette scène mémorable de la réunion des sophistes. Lorsque Socrate s'adresse à Protagoras, le débat s'engage à propos de la possibilité ou non d'enseigner la vertu. Protagoras prétend pouvoir l'enseigner, Socrate reste sceptique ; les positions vont après s'inverser, puisque Socrate soutiendra, vers la fin du dialogue, que les vertus sont des savoirs - et donc peuvent être enseignées -, alors que, pour Protagoras, qui soutient désormais que les vertus sont autre chose que de la science, elles ne peuvent pas être enseignées. Le dialogue débute par le mythe de Prométhée et Epiméthée, au moyen duquel Protagoras expose l'une des rares défenses que nous ayons des procédés délibératifs de l'Athènes démocratique ; de plus, au beau milieu de la discussion philosophique sur l'unité des vertus, nous sommes régalés d'un intermezzo de critique littéraire sur certains vers de Simonide.

Le dialogue est donc complexe; différents thèmes s'entrelacent en une structure littéraire élégante, rendant la lecture de ce dialogue passionnante à plusieurs niveaux. Je voudrais, dans ce petit essai, revisiter les arguments produits par Socrate pour soutenir l'unité des vertus. Je vais laisser de côté toute cette ambiance littéraire pour ne me concentrer que sur certains aspects du quatrième argument, dans la mesure où, de l'aveu même de Socrate, ils aident à mieux comprendre le troisième argument et par là la thèse 
forte de l'unité des vertus. En effet, dans ce dialogue, Socrate avance quatre arguments complets $^{1}$ pour soutenir l'unité des vertus ${ }^{2}$. Les deux premiers fonctionnent de manière indépendante. Dans le premier argument (331a6-332a1), Socrate veut montrer que, parmi toutes les vertus, la justice et la piété, puisqu'elles se ressemblent si fortement homoiotaton, $331 \mathrm{~b} 5$-, semblent bien être l'une telle que l'autre. En fait, cet argument est à comprendre à la lumière de la preuve que Socrate fournit dans l'Euthyphron, à savoir que la piété est une partie de la justice, étant donné que tout acte de piété est un acte juste, mais certains actes justes ne sont pas des actes de piété. De la sorte, la piété devient une partie de la justice et, ainsi, les cinq vertus avec lesquelles on a entamé le dialogue (mentionnées en 330b3-6 : la tempérance, la sagesse, le courage, la justice et la piété) sont reprises, à la fin du dialogue, selon la liste canonique des quatre vertus cardinales : le savoir, la tempérance, le courage et la justice, la piété faisant désormais partie de la justice (voir, par exemple, 361b1-2). Ce n'est pas une mince affaire, car il s'agit ici d'accomplir un rétrécissement du champ sémantique des vertus d'une manière telle qu'on puisse non seulement plaider la cause de leur unité, mais aussi en avoir d'autres conséquences, comme celles que Socrate tire dans la République en vue de la tripartition de l'âme $\mathrm{e}^{3}$. Ce qui m'importe de remarquer ici, c'est le caractère indépendant de ce premier argument : s'il est bon, il opère une prémière identification, sous la forme

1 Je dis quatre arguments complets parce que, à la suite du deuxième argument, Socrate se propose d'enquêter si justice et sagesse (sôphronein) sont identiques ou distinctes, mais cet argument, qui commence en $333 \mathrm{~b} 7$, est interrompu soudainement en 334a2, lorsque Protagoras se met à faire un long discours, ce qui mène à une discussion sur la manière correcte d'entretenir un dialogue, au bout de laquelle Socrate, insatisfait de la décision de Protagoras de ne plus répondre avec des réponses courtes, feint d'abandonner la réunion. C'est ici qui commence le long intermezzo qui porte sur la critique de la poésie de Simonide et qui se clôt en 349 a6 ; à partir de cette ligne, Socrate reprend la discussion sur l'unité des vertus et propose deux nouveaux arguments.

${ }^{2}$ Selon l'article devenu célèbre de G. Vlastos, l'objet de preuve dans ce dialogue ne serait pas l'unité des vertus, mais leur consécution : si l'on en a une, on les a toutes, même si elles diffèrent entre elles en ce qui concerne leur nature (« The unity of the virtues in the "Protagoras" ", publié premièrement dans The Review of Metaphysics, Vol. 25, No. 3 (Mar., 1972), p. 415-458, repris et révisé dans Platonic Studies, Princeton, Princeton University Press, 1973, p. 221-269). La thèse de l'unité des vertus, qui est plus forte que celle de leur consécution (puisqu'elle implique celle-ci, alors que celle-ci ne l'implique pas), a été soutenue de bonne heure : déjà en 1973 T. Penner la soutient selon la perspective de leur unité en tant que discours psychologique («The unity of virtue », dans Philosophical Review, 82 / 1 (1973), p. 35-68). J'ai soutenu la thèse forte de l'unité de toutes vertus en tant que sciences dans Estudos de Ética Antiga, São Paulo, Discurso Editorial, 2007; Paulus, 2009, p. 41-72.

${ }^{3}$ Je renvoie ici à mon texte «Une opération platonicienne : l'avènement des quatre vertus », paru dans Logon didonai - la filosofia come esercizio del render ragione, Studi in onore di Giovanni Casertano, édité par Lidia Palumbo, Casoria, Loffredo, 2011, p. 401-410. 
bien précise d'une réduction de la piété à la justice selon le schéma plus général de la réduction d'une espèce à son genre ${ }^{4}$.

Le deuxième argument, qui se trouve en 332a2-333b5, vise à prouver l'identité entre tempérance et savoir, sophrosune et sophia. Il fait appel à leurs contraires, qui, dans les deux cas, portent le même nom, aphrosune. À partir de là, il est montré ou bien qu'elles sont la même vertu (puisqu'elles ont le même contraire), ou bien qu'un terme unique a plusieurs contraires - en supposant toujours, donc, l'unité du sens d'aphrosune (ce qui est rappelé expressément en $333 \mathrm{~b} 2$ : Évì ővtr). Cet argument mérite une étude à part, mais je ne veux souligner ici que le fait qu'il s'agit encore d'un argument indépendant - s'il est bon, il prouve que sagesse et tempérance sont une seule et même vertu. Il s'ensuit alors un troisième argument, abandonné face à l'agacement croissant de la part de Protagoras. S'il avait été déployé, on aurait eu les identités suivantes : (i) justice $=$ piété ; (ii) tempérance $=$ sagesse; (iii) sagesse $=$ justice, d'où il s'ensuivrait (iv) justice $=$ tempérance, ne manquant qu'une preuve concernant le courage, puisque si l'on démontre que le courage est identique à l'une des trois autres vertus, il est aussitôt démontré que les vertus sont toutes identiques entre elles.

C'est dans ce but que Socrate propose, après le long intermezzo sur les vers de Simonide, un troisième argument (349e1-351b2), grâce auquel il veut montrer que courage et sagesse sont une seule et même vertu. L'argument procède de la manière suivante: on montre d'abord que les hommes courageux sont audacieux pour tout aussitôt montrer que plus on connaît, plus on est audacieux - d'où Socrate veut montrer qu'il existe une connexion intrinsèque entre savoir et courage ${ }^{5}$. Or, alors que Protagoras a admis avoir été réfuté par les deux premiers arguments (ce qui avait provoqué son agacement), il regagne ici confiance, puisqu'il refuse la conclusion tirée par Socrate, en remarquant que, s'il avait concédé à Socrate que tout homme courageux est audacieux, il n'avait pourtant pas admis que tout homme audacieux est courageux. Pour que Socrate puisse obtenir l'identité recherchée entre courage et savoir, il lui faut non

\footnotetext{
${ }^{4}$ Pour cette raison, lorsque Socrate se réfère au résultat de ce premier argument, il ne dit pas que justice et piété sont identiques, mais qu'elles sont « presque la même chose » (333b6).

${ }^{5}$ Ce faisant, Socrate illustre cette connexion intime par le biais de celui qui plonge dans un puits : plus il a la connaissance requise, plus il se montre audacieux en plongeant, et donc avec autant de courage que d'audace. L'usage de cet exemple est, prima facie, opposé à celui qui est fait du même cas dans la première partie du Lachès, où Lachès admet que celui qui plonge sans en avoir la connaissance requise est plus courageux que celui qui y plonge en ayant la connaissance. Beaucoup d'encre a coulé pour expliquer cet usage opposé d'un même exemple, que nous ne pouvons malheureusement pas examiner dans le cadre du présent texte.
} 
seulement affirmer que tout homme courageux est audacieux, mais aussi établir que tout homme audacieux est courageux. Si l'on accepte que celui qui est audacieux est par là même courageux, on admet par là que le savoir opère à l'intérieur du courage, car on avait déjà admis que plus on sait, plus on est audacieux. Pourtant, Protagoras n'avait pas admis que celui qui est audacieux est par là même courageux, et il lui semble bon de ne pas l'admettre, puisque, d'après lui, il y a beaucoup de gens qui, tout en étant audacieux, ne sont pas courageux à proprement parler, bien au contraire.

Le troisième argument opère à l'aide de comparatifs, ce qui le rend plus difficile à manier. Cela dit, je ne veux pas ici tenter de montrer comment on peut le rendre valide, ou, en sens contraire, tenter de montrer où résiderait son échec, selon le soupçon de Protagoras. Ce qui m'importe ici de remarquer, c'est que, au lieu de refaire son argument ou de mieux présenter ses prémisses afin de mettre en évidence qu'il s'agit d'un argument qui aboutit correctement à sa conclusion, Socrate se met tout de suite, et ce de manière fort abrupte, à produire un quatrième argument, celui fondé sur la notion d'acrasie. Cet argument va de $351 \mathrm{~b} 3$ à $358 \mathrm{~d} 4^{6}$. Comme on le sait, dans ce quatrième et dernier argument Socrate montre que la notion même d'acrasie, qui revient à dire qu'on est vaincu soi-même par le plaisir, est une notion incohérente. C'est un argument assez surprenant, et ce à plusieurs égards. Tout d'abord, Socrate ne s'oppose plus à Protagoras, mais les deux unissent leurs forces afin d'enquêter sur la notion d'être vaincu par les plaisirs, notion qui est attribuée à la masse, aux polloi, ceux qui ne sont nullement raffinés et contre qui font bloc désormais Socrate et Protagoras. Ensuite, ce quatrième argument évolue vers un examen de la notion d'hédonisme, mais rien ne nous faisait prévoir une telle discussion, car elle ne semble pas avoir d'incidence sur le problème de l'unité des vertus, sur lequel justement porte ce dialogue. Pourtant, le quatrième argument est expressément produit dans le but de nous montrer dans quel sens le troisième argument était de fait concluant. Cela est dit dès le début, en 353b1-3 : «cet examen nous sera utile pour découvrir le rapport que le courage entretient avec les autres parties de la vertu ». L'application du résultat du quatrième argument pour la solution du troisième se fait de $358 \mathrm{~d} 5$ à $360 \mathrm{e} 5$, et Protagoras se voit enfin forcé d'admettre que le courage aussi se produit en fonction de la connaissance. Le quatrième argument, en dépit de son intérêt intrinsèque à titre de refus socratique de la notion

\footnotetext{
${ }^{6}$ Aristote fera mention de cet argument au tout début de sa propre démonstration concernant la nature de l'acrasie, en le considérant comme une preuve du fait que Socrate niait la possibilité même du phénomène de l'acrasie (EN VII, 3, 1145b22-27).
} 
d'acrasie, est présenté dans le Protagoras dans le but de renforcer le troisième argument, celui qui voulait prouver l'identité entre courage et sagesse, et qui était apparu aux yeux de Protagoras - et sûrement aussi aux yeux de l'audience - comme un échec, un argument qui n'aboutissait pas parce qu'il lui manquait au moins une prémisse (à savoir, que celui qui est audacieux est par là même courageux).

Les deux derniers arguments sont donc soudés dans leur destin, alors que les deux premiers étaient indépendants l'un de l'autre. Mais dans quel sens le quatrième argument (celui consacré à la notion d'acrasie) peut-il nous aider à mieux comprendre le troisième argument (celui sur l'identité entre courage et savoir) ? Sans trop entrer dans les détails, la réponse à cette question me semble être la suivante. Dans le quatrième et dernier argument, Socrate montre que la notion à laquelle s'attache la masse, la notion de plaisir comme étant la source ultime de tout bien, est une notion qui semble - mais ce, uniquement à première vue - exclure tout rapport à la connaissance. Socrate va montrer, au contraire, que la position hédoniste, précisément lorsqu'elle est prise en sa version la plus rigoureuse, non seulement révèle en son intérieur la présence de la raison, sous la forme de l'art de bien mesurer les plaisirs entre eux, mais aussi, ce qui est plus important, le plaisir lui-même, pour qu'il puisse s'exprimer en toute sa puissance, requiert une telle connaissance puisqu'il est gouverné par cet art de la mesure. En effet, l'art de la mesure sert à calculer le plus grand plaisir qu'on puisse avoir selon l'attente raisonnable d'une vie d'une certaine durée. L'art de mesurer devient ainsi la condition même pour qu'on jouisse du plus grand plaisir. Par ce biais, Socrate va donc montrer que, à l'intérieur même du plaisir censé être exempt de toute rationalité et calcul, la raison fait son apparition; plus encore, la raison se montre comme le seul principe selon lequel l'hédoniste rigoureux peut s'assurer du plaisir le plus intense, et ce le plus longuement ${ }^{7}$. Là d'où elle semblait totalement exclue, la raison réapparaît sous la forme de ce qui gouverne la quête même du plaisir. C'est sur ce résultat que Socrate veut s'appuyer pour enfin rendre concluant le troisième

${ }^{7}$ L'hédonisme du Protagoras repose sur deux thèses: (i) tout plaisir est quantifiable en quanta de plaisir et, par là, tout plaisir est commensurable, et (ii) le plaisir est indexé vers l'avenir, car il est structuré en termes d'anticipation et de jouissance future. Ces deux thèses seront modifiées dans le Gorgias, dialogue qui, dans sa troisième et dernière section (celle où Socrate dialogue avec Calliclès), réfléchit sur le plaisir selon deux autres présuppositions: (i) les plaisirs s'organisent selon une hiérarchie qui réflète les préférences qualitatives de l'agent, sans qu'ils soient par là quantifiables en quanta commensurables, et (ii) le plaisir se jouit dans le présent, il ne se projette sur l'avenir que pour se reproduire dans une sorte de présent prolongé indéfiniment. Les arguments socratiques contre l'hédonisme du Protagoras ne servent donc plus à répondre à l'hédonisme callicléen, puisque les présuppositions de ce dernier sont fort distinctes de celles de l'hédonisme protagoréen, et Socrate doit donc nous fournir de nouveaux arguments pour réfuter philosophiquement l'hédonisme callicléen. 
argument. Les courageux sont audacieux, et sont davantage audacieux en fonction du savoir dont ils disposent. Là aussi donc, d'où le savoir semblait par excellence exclu du courage, qui, selon Protagoras, est la seule vertu qu'on peut exercer alors qu'on ne dispose d'aucune connaissance $(349 \mathrm{~d} 2-8)$ - il est montré, au moyen de la notion d'audace, que le courage en tant que vertu se met en place en intime connexion avec le savoir : plus on sait, plus on est audacieux; plus on est audacieux, plus on devient courageux.

À ceci près que Socrate semble supposer - ce qu'il ne démontre nulle part dans ce dialogue, ou du moins jamais expressément - que la véritable audace se décline en rapport direct avec la connaissance, selon une échelle bien précise : celui qui sait le plus est le plus audacieux. Il existe bien entendu quelque chose qui ressemble à l'audace, un genre de témérité que même les ignorants peuvent exercer. Cette témérité n'est pourtant pas de la véritable audace, ou mieux : elle n'est pas de l'audace du tout. Elle lui ressemble, certes, mais cette ressemblance est trompeuse, car la véritable audace dépend étroitement de la détermination correcte de l'objet à craindre ou à fuir, et cette détermination correcte n'est possible qu'en fonction du savoir dont dispose l'agent proprement courageux (359e1-360e5). Protagoras emploie de manière incorrecte le terme d'audace en se référant à des cas qui, à proprement parler, ne sont pas du tout des cas d'audace, même s'ils lui ressemblent. Prodicos, qui assiste à toute la discussion et qui est toujours présenté par Platon comme étant très friand de distinctions sémantiques, ne semble pas non plus se rendre compte de cette grammaire philosophique de l'audace et du courage ${ }^{8}$. Pourtant Socrate réserve l'usage d'audace uniquement aux cas d'audace véritable, celle qui est coordonnée avec le savoir. Creusant le même sillon, Socrate va montrer, dans le quatrième argument, que la masse utilise, bien fréquemment, la notion d'être vaincu par le plaisir, alors que, selon la grammaire philosophique, une telle expression est tout bonnement incohérente au sein d'une perspective hédoniste rigoureuse. C'est cette leçon qui est donnée en sourdine grâce au quatrième argument et qui nous permet de revisiter le troisième argument de façon à finalement comprendre pourquoi il est concluant: l'audace (véritable) se fait en stricte dépendance de la connaissance, et par là courage et savoir peuvent s'identifier, alors que, comme

\footnotetext{
${ }^{8}$ Prodicos se contente, en fait, de remarquer, à propos de la définition avancée par Socrate de crainte ou

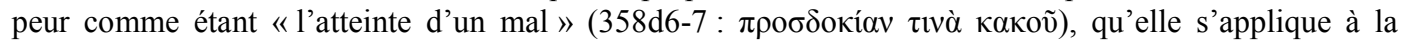

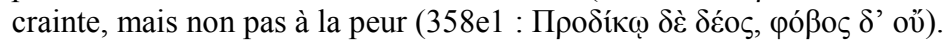


Protagoras le supposait encore, il paraissait - mais faussement - qu'on pouvait être courageux sans avoir aucune connaissance (des choses à craindre ou à fuir) ${ }^{9}$.

Pour atteindre son but, Socrate doit partir, dans le quatrième argument, d'une position hédoniste radicale, c'est-à-dire, doit proposer comme thèse hédoniste centrale l'identité tout court entre plaisir et bien suprême, l'identité pleine entre jouissance et bonheur, selon laquelle une chose est bonne parce qu'elle nous apporte du plaisir et de la jouissance. C'est le cas limite, celui où tout calcul et raisonnement semblent disparaitre du bonheur, car celui-ci n'est alors que l'expression de l'agréable et de la simple jouissance. En outre, Socrate doit montrer que même si elle ne s'en tient pas forcément à une notion précise du plaisir comme étant la source ultime de toutes nos motivations pour agir, la masse la présuppose néanmoins. Il incombe donc au philosophe, et non pas à la masse, d'affirmer d'une part l'identité forte entre plaisir et bien en tant que version rigoureuse de l'hédonisme, et d'autre part de montrer que les polloi adhèrent à cette version rigoureuse du plaisir en tant que source ultime et unique de nos motivations, en dépit de leur langage vague et hésitant. C'est exactement ce que fait Socrate, me semble-t-il, en 351c4-5 et 352b5, respectivement.

Examinons premièrement l'identité tout court entre plaisir et bien. Voici le texte grec :

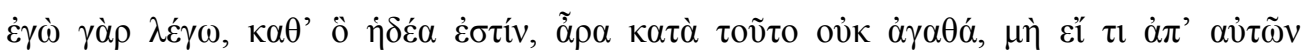

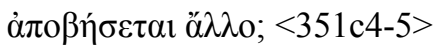

C'est le texte que donne Burnet ${ }^{10}$, d'après les mss. B T W. Le sens de $\mu$ ̀̀ cì est bien rendu par les traductions françaises : «quoi qu'il puisse en sortir d'ailleurs » $\left(\right.$ Croiset $\left.^{11}\right)$, «abstraction faite de ce qui peut en résulter par ailleurs » (Ildefonse ${ }^{12}$ ). Le point est que Socrate propose une thèse selon laquelle l'identification entre bien et plaisir se fait sans aucune restriction : une chose est bonne dans la mesure où elle est agréable, point à la ligne. Elle peut avoir d'autres conséquences, comme celle de nuire à la santé, mais cela n'est pas en cause lorsqu'on la détermine en tant que bonne, puisque l'être bon pour une chose ne dépend que du fait que cette chose est agréable, toute autre

${ }^{9}$ On retrouvera aussi chez Aristote le thème des formes de comportement qui ressemblent au courage, mais qui ne sont point de vrai courage, c'est-à-dire ne sont pas du tout du courage : voir à cet égard Éthique à Nicomaque III, 11, 1116a15-1117a28.

${ }^{10}$ Voir John Burnet, Platonis Opera - tomus III, Oxford, Clarendon Press 1992 (1e éd. 1903).

${ }^{11}$ Voir Platon, Protagoras, éd. Maurice Croiset, Paris, Les Belles Lettres, 1984

${ }^{12}$ Voir Platon, Protagoras, trad. Frédérique Ildefonse, Paris, GF Flammarion, 1997. 
considération n'ayant aucune incidence là-dessus. Peut-être ne vivra-t-on qu'une journée si l'on jouit d'un tel plaisir, dont l'intensité serait en étroit rapport avec une très courte existence - mais si bien vivre consiste justement en jouir du plaisir, que l'on jouisse du plaisir le plus intense, au diable la durée de la vie ! La seconde main de T propose pourtant un texte différent :

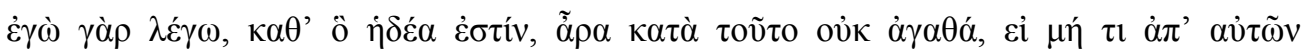

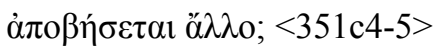

La traduction américaine de Stanley Lombardo et Karen Bell suit ce texte : « if it results in nothing other than pleasure $» .{ }^{13}$ Cela veut dire que, à moins qu'il y ait un résultat différent, par exemple si la conséquence est nuisible pour la santé, la chose agréable est prise comme bonne. Mais cette condition n'est pas en question ici ; Socrate veut, au contraire, proposer la thèse forte de la réduction de ce qui est bon à ce qui est agréable, sans aucune restriction, parce qu'il veut montrer que, même dans sa version la plus radicale, l'art de mesurer y fera son apparition comme étant l'élément décisif pour la détermination du plus grand plaisir (ou encore, dans les termes de cette thèse, du plus grand bonheur ${ }^{14}$. Le problème du passage se transfère alors vers le début de la phrase,

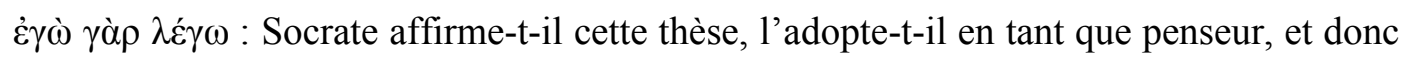
soutient-il l'hédonisme, ou bien ne fait-il qu'expliciter le sens qu'on doit donner à la thèse hédoniste, sans par là l'affirmer ou l'adopter en son propre nom ? Il me semble que toute la stratégie de l'argument plaide fortement pour un Socrate qui explicite la thèse que lui et Protagoras doivent désormais mettre à l'épreuve, qui n'est sûrement pas la thèse que Socrate aurait soutenue en son propre nom, loin de là ! Le débat pourtant,

\footnotetext{
${ }^{13}$ Voir Stanley Lombardo \& Karen Bell, Protagoras, in Cooper, John M. (ed.) Plato - Complete Works, Indianapolis, Hackett Publishing Company 1997. La traduction de Christopher Charles Whiston Taylor, «leaving their other consequences out of account», me semble rester ambiguë, car elle peut dire soit qu'on n'a pas à s'inquiéter de quoi que ce soit qui puisse en découler, mais uniquement du plaisir dont on jouit, soit qu'il faudra quand même ajouter à la simple jouissance l'analyse de ses conséquences dans l'avenir (voir Christopher Charles Whiston Taylor, Plato's Protagoras, Oxford, Clarendon Press, 1976).

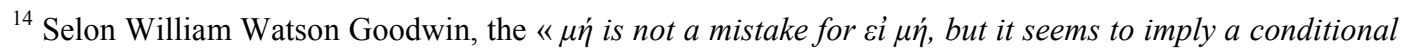

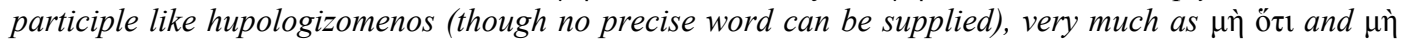
ö $\pi \omega \varsigma$ imply a verb of saying (707). The meaning clearly is, Are not things good just so far as they are pleasant, if we take no account of any other (i.e. unpleasant) element in them? This sense would hardly be found in the emended reading ei $\mu$ í $\tau \mathrm{l}$ » (voir Syntax of the moods and tenses of the Greek verb. London, Macmillan, 1897, p. 176-177). Cela est vrai si l'explication qu'on veut donner doit préserver la possibilité d'attribuer cette thèse à Socrate. Si, par contre, Socrate n'adopte aucunement cette thèse hédoniste, mais

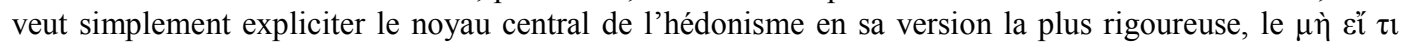
reprend son sens de "toute autre considération étant mise de côté », "peu importe toute autre conséquence » ou encore : « au diable avec les conséquences ! », car elles n'ont aucune emprise sur ce qui fait qu'une chose soit bonne, à savoir : le fait tout simplement de nous procurer du plaisir. Je remercie Daniel Lopes d'avoir attiré mon attention sur cette remarque de Goodwin.
} 
comme on le sait, fait encore rage. Pour reprendre les mots d'un des ténors de la position opposée :

Socrates here makes an explicit assertion of his own view, in the form of a rhetorical question ${ }^{15}$.

Un signe qu'il ne s'agit pas de la position de Socrate lui-même, mais d'une thèse qu'il explicite dans sa version la plus rigoureuse afin de mieux la mettre à l'épreuve, est le fait que, un peu plus loin, en 354e8-355a2, lorsqu'il revient sur l'identification entre bien et plaisir (au profit de ce dernier), Socrate permet pour une dernière fois, avant de mener à bout sa réfutation, que la masse retire son identification entre bien et plaisir et qu'elle propose à sa place une autre opinion sur les rapports entre ce qui est bon et ce

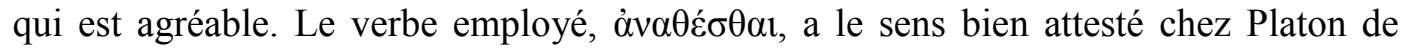
retirer ce qu'on a avancé, comme dans le jeu de dames. Mais la thèse n'est pas retirée, et Socrate procède directement à sa réfutation. La vraie question me semble être la suivante : Socrate peut-il vraiment attribuer à la masse une thèse aussi forte, la quête du

${ }^{15}$ Christopher Charles Whiston Taylor, in Plato, Protagoras, trad. cit., 1991, p. 166. Puisqu'il attribue

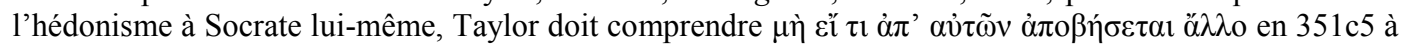
titre d'une qualification nécessaire pour la position socratique, «since Socrates does not deny that some pleasant activities and experiences are in fact bad (cf. 353c-354e), but merely maintains that such things are bad, not in themselves but in so far as they have bad (i.e. unpleasant) consequences " (ibidem, p. 167). Indépendamment de savoir si une telle position est cohérente ou non, le point consiste à déterminer si Socrate exprime sa propre position, ou simplement s'il met à l'épreuve une position qu'il attribue à d'autres personnes, le cas échéant à la masse. Déjà C. C. W. Taylor et J. Gosling attribuaient à Socrate l'hédonisme dans le Protagoras: «we suggest, then, that we have sufficient evidence to render at least probable the hypothesis that in writing the Protagoras Plato presented what he took to be Socrates' actual view of the nature of goodness, viz. that goodness is identical with long-term pleasantness " (voir Justin Cyril Bertrand Gosling et Christopher Charles Whiston Taylor, The Greeks on Pleasure, Oxford, Clarendon Press, 1984, p. 67). Terence Irwin aussi l'attribue à Socrate, tout en soulignant que, dans le Protagoras, Socrate «affirms epistemological hedonism, taking judgements about pleasure to be epistemologically prior to judgements about goodness » (voir Terence Irwin, Plato's Ethics, New York / Oxford, Oxford University Press, 1995, p. 83). La lecture que je propose est aux antipodes d'une telle attribution : le Socrate de Platon n'a jamais entretenu une thèse hédoniste par rapport au bonheur, et lui attribuer la version que Taylor propose de l'hédonisme (ou celle révisée d'un hédonisme épistémologique) revient à lui attribuer précisément la position que Socrate va réfuter dans le quatrième argument, lorsqu'il montre que le plaisir requiert un autre élément, le savoir, pour qu'il puisse rayonner selon l'art de la mesure. D'ailleurs, Protagoras n'adhère pas non plus à une telle thèse. En fait, il ne

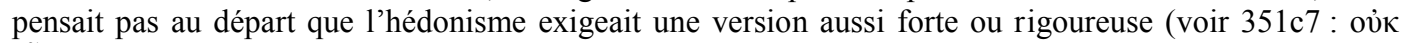
oĩ $\delta \alpha$, «je ne sais pas »); ce n'est qu'après, en réfléchissant avec Socrate, qu'il la lui accorde, et prend immédiatement toutes ses distances vis-à-vis d'une telle thèse. La masse elle non plus n'y adhère pas : le passage 351c2-3 montre assez clairement que la masse n'entretient pas une telle thèse, au moins non explicitement, tout en s'adonnant pourtant à la quête effrenée du plaisir. En effet, il revient au philosophe de formuler la version la plus rigoureuse et exigeante de l'hédonisme afin de le mettre à l'épreuve, et c'est exactement ce que Socrate fait en 351c4-5 et qu'il reprend en 351e2-3, en compagnie de Protagoras contre ce que la masse doit accepter. Il est important aussi de remarquer que, selon l'analyse proposée par Socrate, la raison refait surface au beau milieu du plus rigoureux hédonisme, mais elle reste une raison pragmatique et subordonnée à la quête du plaisir - une thèse que Platon n'a jamais, dans aucun de ses dialogues, attribuée à Socrate. 
plaisir sans aucun égard pour d'autres conséquences, en réduisant la notion de bien à celle de jouissance?

Il me semble que Socrate peut la lui attribuer, dans la mesure où il dégage cette thèse en tant que philosophe de ce que les polloi disent d'une manière plutôt confuse. Ce n'est pas une thèse que la masse entretient ouvertement, mais c'est la thèse qu'impliquent les positions adoptées par la masse. Autrement dit: la masse suit l'hédonisme, et voici la version la plus rigoureuse de l'hédonisme : ce qui est bon est réduit à ce qui est agréable, point à la ligne. Une chose est bonne parce qu'elle nous procure du plaisir, au diable les conséquences !

Examinons maintenant le passage 352b3-c2, où Socrate mentionne ce que disent les hommes à propos de ce qui nous motive à agir :

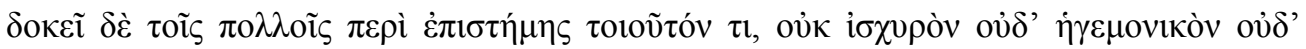

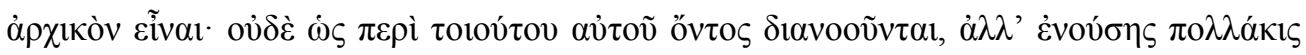

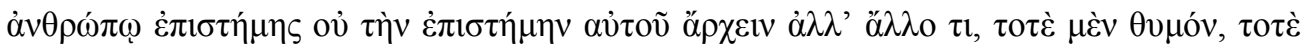

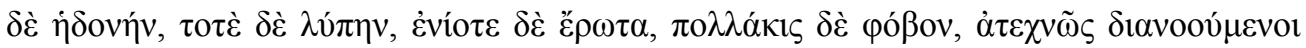

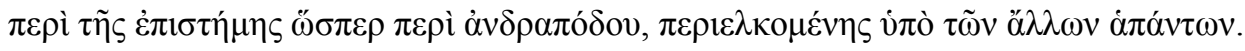

C'est le texte que donne Burnet. Voici une traduction représentative de ce qu'on lit traditionnellement dans ce passage :

Most people think this way about it $<$ scl. science $>$, that it is not a powerful thing, neither a leader nor a ruler. They do not think of it in that way at all; but rather in this way: while knowledge is often present in a man, what rules him is not knowledge but rather anything else - sometimes anger, sometimes pleasure, sometimes pain, at other times love, often fear; they think of his knowledge as being utterly dragged around by all these other things, as if it were a slave ${ }^{16}$.

Et voici le commentaire que Denyer ajoute à ce passage :

Socrates could add to this list those impulses to sneeze or yawn or weep or giggle which we know we could and should resist but to which we nevertheless succumb. Moreover, even as it stands, the list includes too many motivations to fit well with the argument ahead. For Socrates will argue that pleasure (352d4-356c3) and fear (358d6-360d9) cannot stop us doing what we know we should and can; and none of his arguments will even mention anger or love; yet he will proceed as if his arguments have shown that knowledge cannot be overcome by any rival motivation whatsoever ${ }^{17}$.

Il est en effet trop difficile de ne pas bâiller, même quand on sait qu'il faut éviter tout bâillement. Mais les bâillements n'appartiennent pas à cette liste ; il ne s'agit pas de mentionner des actes involontaires, que le savoir ne contrôle pas, mais de dresser une

\footnotetext{
${ }^{16}$ Plato, Protagoras, trad. Stanley Lombardo \& Karen Bell, op. cit., p. 782.

${ }^{17}$ Nicholas Denyer, Plato's Protagoras, Cambridge, Cambridge University Press, 2008, p. 180.
} 
liste (non exhaustive) des sources de motivations qui, selon la masse, battent en brèche le savoir, ce qu'un bâillement ne peut que rarement produire (comme lorsqu'on veut, par exemple, donner signe de notre ennui, alors qu'on sait qu'on ne doit pas l'afficher).

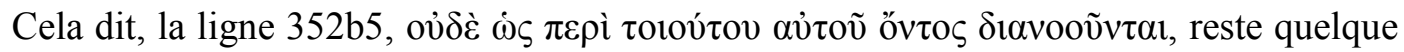
peu surprenante, car elle ne fait que répéter ce qui vient tout juste d'être dit. Cela est possible mais éveille le soupçon qu'il y a peut-être quelque chose de plus ici. En fait, la version que nous donne Burnet provient d'un mélange de deux traditions des manuscrits. D'après l'apparat critique de Burnet, on aurait les deux versions suivantes :

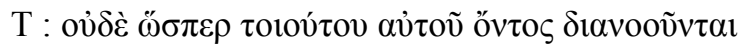

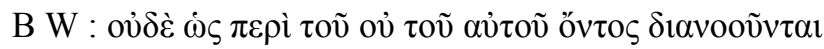

Je vais laisser de côté la version $\mathrm{T}$, car elle donne le même résultat que celle proposée par Burnet. Par contre, B W présentent un texte bien différent. En effet, en

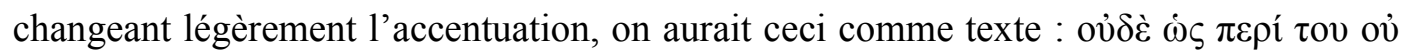

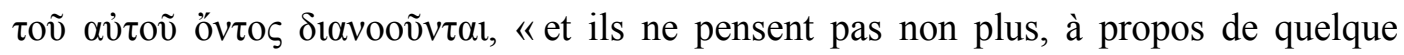
chose, comme étant la même chose $<$ qui commande $>{ }^{18}$. En adoptant cette dernière version réaccentuée, on a ceci comme traduction pour tout ce passage :

L'avis des gens à propos de la science, c'est à peu près ceci, qu'elle n'a pas de force, ne commande pas, ne gouverne pas ; ils ne pensent pas non plus, à propos de quelque chose, comme étant la même chose <qui commande>, mais que, souvent, la science étant présente dans l'homme, ce n'est pas elle qui commande en lui, mais autre chose : tantôt la fougue, tantôt le plaisir, tantôt la peine, quelquefois l'amour, souvent la crainte, ce qui fait qu'ils pensent la science comme si c'était un esclave tiraillé par toutes ces autres choses. $(352 \mathrm{~b} 3-\mathrm{c} 2)$

La version B W est tout à fait intéressante, car on n'a plus la répétition de l'idée que la science ne commande pas; au contraire, Socrate nous dit que, d'après ce que croit la masse, ce n'est pas la même chose qui s'impose à la science et qui la domine,

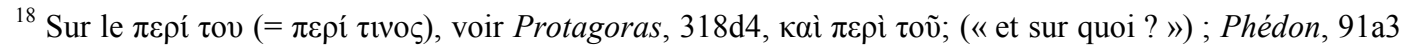

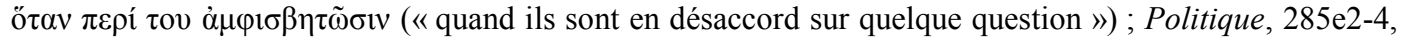

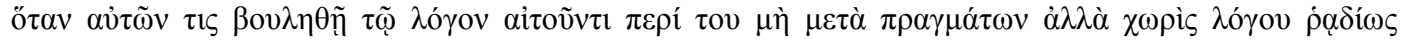
$\dot{\varepsilon} v \delta \varepsilon \dot{\xi} \xi \alpha \sigma \theta \alpha$ l (« quand on souhaite, à qui sollicite une explication sur quelque chose, la tirer au clair non

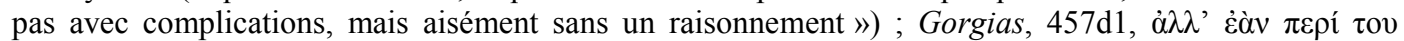

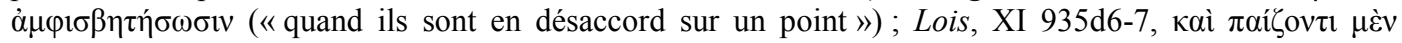

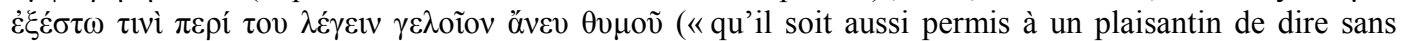

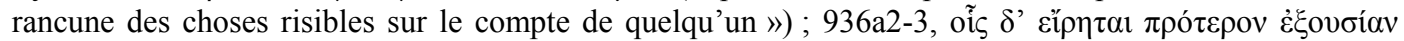

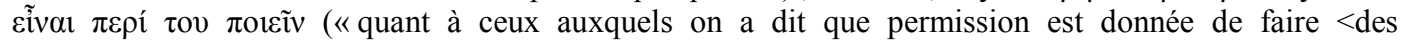
compositions comiques $>$ sur le compte de quelqu'un »). Pour la double négation ov̉ $\delta \dot{\varepsilon}$... ov่, c'est une

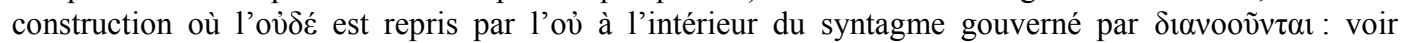

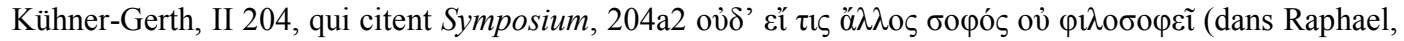
Blass, Friedrich, Wihelm Kühner \& Bernhard Gerth, Ausführliche Grammatik der Griechischen Sprache, Hanover, Hahnsche Buchhandlung, 1890, et Herbert Weir Smyth Greek Grammar, Cambridge MA, Harvard University Press, 1920, réimp. 1984, par. 2940.
} 
mais c'est tantôt ceci, tantôt cela; parfois ceci, parfois cela. Il nous est naturel à nous de supposer que tout ce qui domine la science se réduit en fait au plaisir et à la jouissance mais cela n'est que ce que le philosophe nous apprend lorsqu'il examine la manière dont parle la masse à ce propos, tandis que pour la masse ce n'est pas toujours la même chose qui domine le savoir. C'est donc Socrate qui va attribuer, à la suite du texte, cette thèse à la masse : le plaisir et son contraire, la peine, sont les notions centrales quand on affirme que la science est tiraillée de tous côtés. À partir de là, et après avoir formulé l'hédonisme selon sa version la plus rigoureuse (la thèse forte selon laquelle le bien n'est autre chose que l'agréable, point à la ligne : une chose est bonne parce qu'elle nous procure de la jouissance), Socrate entame sa réfutation coutumière. Le quatrième argument se met alors en place; par ce dispositif, on verra le savoir refaire surface derrière le plaisir sous la forme de l'art de mesurer, en sorte que, même dans un domaine à première vue totalement hostile à ses prétentions, la science marque de son empreinte la quête effrenée du plaisir - même si, il faut l'avouer, le savoir lui reste subordonné, puisqu'il ne fait que garantir à l'hédoniste le plus intense et le plus long plaisir. Néanmoins, on est par là censé mieux comprendre comment le fait que l'audace se décline en fonction du savoir nous permet de conclure que le courage et la science sont une seule et même vertu : là aussi, dans un domaine qui semblait prima facie hostile à tout raisonnement, le calcul et la raison refont surface, puisqu'ils sont ce qui détermine ce qui est l'objet véritable de crainte ; et, de ce fait, la connaissance se remet en place, en tant que ce qui détermine l'acte véritable d'audace, ou mieux : ce qui caractérise l'acte d'audace tout simplement (à condition, bien entendu, qu'on suive la grammaire philosophique de ce terme, sans lui accorder un usage trop large et donc trompeur).

\section{Bibliographie}

Burnet John, Platonis Opera - tomus III, Oxford, Clarendon Press, 1903, réimp.1992.

Croiset Maurice, Platon : Protagoras, Paris, Les Belles Lettres, 1984.

Denyer Nicholas, Plato's Protagoras, Cambridge, Cambridge University Press, 2008.

Goodwin William Watson, Syntax of the moods and tenses of the Greek verb. London, Macmillan, 1897.

Gosling Justin Cyril Bertrand and Taylor Christopher Charles Whiston, The Greeks on Pleasure, Oxford, Clarendon Press, 1984.

Ildefonse Frédérique, Platon : Protagoras, Paris, GF Flammarion, 1997. 
Irwin Terence, Plato's Ethics, New York ; Oxford, Oxford University Press, 1995.

Kühner Raphael, Blass Friedrich Wihelm \& Bernhard Gerth, Ausführliche Grammatik der Griechischen Sprache, Hanover, Hahnsche Buchhandlung, 1890.

Lombardo Stanley \& Karen Bell, Protagoras, in John M. Cooper, (ed.) Plato - Complete Works, Indianapolis, Hackett Publishing Company, 1997.

Penner Terry, «The unity of Virtue », in Philosophical Review 82 / 1 (1973), p. 35-68.

Smyth Herbert Weir, Greek Grammar, Cambridge MA, Harvard University Press, 1984 (1920).

Taylor Christopher Charles Whiston, Plato's Protagoras, Oxford, Clarendon Press, 1976.

Vlastos Gregory, Platonic Studies, Princeton, Princeton University Press, 1973.

Zingano Marco, «Une opération platonicienne : l'avènement des quatre vertus », dans Lidia Palumbo (éd.), Logon didonai - la filosofia come esercizio del render ragione, Studi in onore di Giovanni Casertano, Casoria, Loffredo, 2011.

Zingano Marco, Estudos de Ética Antiga, São Paulo, Discurso Editorial, 2007; Paulus, 2009. 